\title{
Temozolomide and Radiotherapy versus Radiotherapy Alone in High Grade Gliomas: A Very Long Term Comparative Study and Literature Review
}

\author{
Salvatore Parisi, ${ }^{1,2}$ Pietro Corsa, ${ }^{1}$ Arcangela Raguso, ${ }^{1}$ Antonio Perrone, \\ Sabrina Cossa, ${ }^{1}$ Tindara Munafò, ${ }^{1}$ Gerardo Sanpaolo, ${ }^{1}$ Elisa Donno, ${ }^{1}$ \\ Maria Antonietta Clemente, ${ }^{1}$ Michele Piombino, ${ }^{3}$ Federico Parisi, ${ }^{4}$ and Guido Valle ${ }^{5}$ \\ ${ }^{1}$ Unit of Radiation Therapy of IRCCS “Casa Sollievo della Sofferenza”, 71013 San Giovanni Rotondo, Italy \\ ${ }^{2}$ Radiotherapy Unit, Scientific Institute "Casa Sollievo della Sofferenza”, Viale Cappuccini, 71013 San Giovanni Rotondo, Italy \\ ${ }^{3}$ Unit of Radiation Therapy of Policlinico, 70124 Bari, Italy \\ ${ }^{4}$ University of Chieti, 66100 Chieti, Italy \\ ${ }^{5}$ Unit of Nuclear Medicine of IRCCS “Casa Sollievo della Sofferenza”, San Giovanni Rotondo, Italy
}

Correspondence should be addressed to Salvatore Parisi; s.parisi@operapadrepio.it

Received 7 July 2014; Revised 4 November 2014; Accepted 5 November 2014

Academic Editor: Chi-Feng Hung

Copyright (c) 2015 Salvatore Parisi et al. This is an open access article distributed under the Creative Commons Attribution License, which permits unrestricted use, distribution, and reproduction in any medium, provided the original work is properly cited.

Temozolomide (TMZ) is the first line drug in the care of high grade gliomas. The combined treatment of TMZ plus radiotherapy is more effective in the care of brain gliomas then radiotherapy alone. Aim of this report is a survival comparison, on a long time ( $>10$ years) span, of glioma patients treated with radiotherapy alone and with radiotherapy + TMZ. Materials and Methods. In this report we retrospectively reviewed the outcome of 128 consecutive pts with diagnosis of high grade gliomas referred to our institutions from April 1994 to November 2001. The first 64 pts were treated with RT alone and the other 64 with a combination of RT and adjuvant or concomitant TMZ. Results. Grade 3 (G3) haematological toxicity was recorded in 6 (9\%) of 64 pts treated with RT and TMZ. No G4 haematological toxicity was observed. Age, histology, and administration of TMZ were statistically significant prognostic factors associated with 2 years overall survival (OS). PFS was for GBM 9 months, for AA 11. Conclusions. The combination of RT and TMZ improves long term survival in glioma patients. Our results confirm the superiority of the combination on a long time basis.

\section{Introduction}

Despite advances in the last years in the treatment of neoplastic diseases, the prognosis of patients (pts) with high grade gliomas is still dismal.

The survival of glioma patients treated with surgical resection alone is approximately 6 months [1]. The combination of surgery and postoperative radiation therapy (RT) increases the survival up to 9-10 months in pts with glioblastoma multiforme (GBM) and 36 months in anaplastic astrocytoma (AA) [2].

In order to get further improvements in the last decades many studies have tested multimodality treatment schedules incorporating chemotherapy (CT) with nitrosourea based regimens, with questionable survival advantages [1-6].
A meta-analysis published in 2002 [7] including many different chemotherapeutic regimes has pointed out that the association of chemotherapy is, in general, more effective than RT alone in prolonging survival and in delaying recurrences in glioma patients. Particularly this meta-analysis showed a mild but significant benefit with the addition of CT, with a $15 \%$ relative reduction in the risk of death and an increase in 2-year survival from $9 \%$ to $13 \%$ in individuals with GBM and from $31 \%$ to $37 \%$ in pts with AA. These evidences encouraged research with new chemotherapeutic agents.

Temozolomide (TMZ) (Temodal, Temodar; ScheringPlough, Kenilworth, NJ) is one of second-generation imidazotetrazinone prodrugs that spontaneously converts into the active metabolite without the need for enzymatic demethylation in the liver [8]. Nowadays TMZ is the first choice drug in 
TABLE 1: Group A and group B: patients' characteristics.

\begin{tabular}{|c|c|c|}
\hline & Group A & Group B \\
\hline Patients' number & 31 & 33 \\
\hline \multicolumn{3}{|l|}{ Age } \\
\hline Range & 41-78 years & $26-74$ years \\
\hline Median & 62 years & 57 years \\
\hline \multicolumn{3}{|l|}{ Sex } \\
\hline Male & 17 pts & $18 \mathrm{pts}$ \\
\hline Female & 14 pts & $15 \mathrm{pts}$ \\
\hline \multicolumn{3}{|l|}{ Karnofsky Index } \\
\hline Range & $60-90$ & $60-90$ \\
\hline Medium & 70 & 70 \\
\hline \multicolumn{3}{|l|}{ Surgery } \\
\hline Stereotactic biopsy & 3 pts & 9 pts \\
\hline Subtotal resection & 18 pts & $12 \mathrm{pts}$ \\
\hline Total resection & $10 \mathrm{pts}$ & 12 pts \\
\hline \multicolumn{3}{|l|}{ Histology } \\
\hline Anaplastic astrocytoma & $11 \mathrm{pts}$ & 10 pts \\
\hline Glioblastoma multiforme & 20 pts & $23 \mathrm{pts}$ \\
\hline \multicolumn{3}{|l|}{ RT total dose } \\
\hline Median & $64 \mathrm{~Gy}$ & $63 \mathrm{~Gy}$ \\
\hline Range & 45 Gy-66 Gy & $45 \mathrm{~Gy}-64 \mathrm{~Gy}$ \\
\hline$<50$ Gy & $2 \mathrm{pts}$ & $1 \mathrm{pts}$ \\
\hline$\geq 50$ Gy $<60$ Gy & $5 \mathrm{pts}$ & 2 pts \\
\hline$\geq 60 \mathrm{~Gy}$ & 24 pts & 30 pts \\
\hline
\end{tabular}

the chemotherapy of gliomas and is largely used after surgery and together or after RT.

Accordingly, 64 consecutive pts, with diagnosis of high grade glioma, with irradiation and adjuvant TMZ (group A, $n=31 \mathrm{pts}$ ) or adjuvant/concomitant (group B, $n=33 \mathrm{pts}$ ) [9] were treated at the Departments of Radiotherapy of Bari University and "Casa Sollievo della Sofferenza" Hospital in San Giovanni Rotondo. The survival data of these subjects (groups $\mathrm{A}+\mathrm{B}$ ) were compared with a group of 64 other patients with similar clinical characteristics treated in the same institutions only with radiotherapy from April 1994 to December 1996 (group C).

This study is aimed at comparing the outcome of the 31 pts treated with RT and adjuvant TMZ (group A) from January 1997 to June 1999 versus the 33 pts treated with RT and concomitant TMZ (group B) from July 1999 to November 2001 and at comparing, on a long term basis, the subjects that received both RT and TMZ with the historical group (group C) that was treated with radiotherapy alone. The survival data have been evaluated also according to the histology of the neoplasm: glioblastoma multiforme (GBM, in 43 patients of groups $A+B$ and in 50 pts of group $C$ ) and anaplastic astrocytoma (AA, in 21 pts of groups $\mathrm{A}+\mathrm{B}$ and in 14 pts of group C).

\section{Patients and Methods}

2.1. Inclusion Criteria. Our retrospective analysis included pts aged $>18$ years with pathologically proven diagnosis of AA
TABLE 2: Groups A + B and group C: patients' characteristics.

\begin{tabular}{|c|c|c|}
\hline & Groups A + B & Group C \\
\hline Patients' number & 64 & 64 \\
\hline \multicolumn{3}{|l|}{ Age } \\
\hline Range & $26-78$ years & 29-74 years \\
\hline Median & 59 years & 60 years \\
\hline \multicolumn{3}{|l|}{ Sex } \\
\hline Male & 35 pts & 39 pts \\
\hline Female & 29 pts & $25 \mathrm{pts}$ \\
\hline \multicolumn{3}{|l|}{ Karnofsky Index } \\
\hline Range & $60-90$ & $60-90$ \\
\hline Medium & 70 & 70 \\
\hline \multicolumn{3}{|l|}{ Surgery } \\
\hline Stereotactic biopsy & 12 pts & $11 \mathrm{pts}$ \\
\hline Subtotal resection & 30 pts & 35 pts \\
\hline Total resection & 22 pts & $18 \mathrm{pts}$ \\
\hline \multicolumn{3}{|l|}{ Histology } \\
\hline Anaplastic astrocytoma & $21 \mathrm{pts}$ & $14 \mathrm{pts}$ \\
\hline Glioblastoma multiforme & 43 pts & 50 pts \\
\hline \multicolumn{3}{|l|}{ RT total dose } \\
\hline Median & $61 \mathrm{~Gy}$ & $59 \mathrm{~Gy}$ \\
\hline Range & 45 Gy-66 Gy & $35 \mathrm{~Gy}-66 \mathrm{~Gy}$ \\
\hline$<50$ Gy & 3 pts & 3 pts \\
\hline$\geq 50$ Gy $<60$ Gy & $7 \mathrm{pts}$ & 6 pts \\
\hline$\geq 60 \mathrm{~Gy}$ & 54 pts & $55 \mathrm{pts}$ \\
\hline
\end{tabular}

or GBM. All histologic specimens were classified according to World Health Organisation (WHO) criteria, after surgery or stereotactic biopsy. Other inclusion criteria were a Karnofsky Index (KI) of 60-100, normal haematological, renal, and hepatic functions, absence of previous (with the exception of nonmelanoma skin cancer and carcinoma in situ of the cervix) or concurrent neoplasm, and absence of any other remarkable disease.

2.2. Patients' Characteristics. The study refers to 64 consecutive pts referred to our Departments of Radiotherapy that started brain neoplasm treatment from January 1997 to November 2001.

Out of 64 pts, 29 were females and 35 males, with age ranging from 26 to 78 years, with a median of 59 years. In 12 pts, with inoperable diseases, only a stereotactic biopsy was performed and in 52 pts a surgical resection was performed (30 subtotal and 22 total) (Tables 1 and 2 ).

The histology of the neoplasm was glioblastoma multiforme (GBM, in 43 patients of groups $A+B)$ and anaplastic astrocytoma (AA, in 21 pts of groups $\mathrm{A}+\mathrm{B}$ ). The control group histology (group C) included 50 pts with GBM and 14 pts with AA.

2.3. Treatment. The $64 \mathrm{pts}$ of groups $\mathrm{A}+\mathrm{B}$ were treated with RT and oral TMZ. Median total dose of RT delivered was 63.5 Gy (range $45 \mathrm{~Gy}-66 \mathrm{~Gy}$ ), with conventional fractionation, according to ICRU recommendations (Table 1). Ten pts 
were treated with a total dose less than $60 \mathrm{~Gy}$ because of their low (60 to 75$) \mathrm{KI}$ or disease progression during the treatment.

Irradiation volume was determined on preoperative computed tomography (CT) and magnetic resonance (MR) of the brain and planning target volume (PTV) included the neoplasm, the surrounding oedema, and a margin of $2 \mathrm{~cm}$ in all directions [10]. All pts were immobilised with a customised thermoplastic mask. Three-dimensional treatment planning was obtained on the basis of CT performed with pt immobilised in therapy position.

The first 31 pts from January 1997 to June 1999 (group A) were treated with RT and adjuvant TMZ $\left(200 \mathrm{mg} / \mathrm{m}^{2} / \mathrm{d} \times 5\right.$ days, every 28 days for 6 cycles) and the other 33 from July 1999 to November 2001 (group B) with RT and concomitant TMZ ( $75 \mathrm{mg} / \mathrm{m}^{2} / \mathrm{d} \times 7 \mathrm{~d} /$ wk for 6 weeks) followed by adjuvant TMZ (200 $\mathrm{mg} / \mathrm{m}^{2} / \mathrm{d} \times 5$ days, every 28 days for 5-6 cycles).

During the concomitant and adjuvant radiochemotherapeutic regimens, prophylactic antiemetic therapy (Ondansetron $8 \mathrm{mg}$ /die or Granisetron $2 \mathrm{mg} / \mathrm{die}$ ) was routinely prescribed. Anticonvulsant and corticosteroids were used only as required.

The results obtained in the 31 pts of group A have been compared with those of the 33 of group B. Finally we have compared the results obtained in these 64 pts (group $\mathrm{A}+$ group B) with those of a historical group of 64 consecutive pts (group C), with similar clinical characteristics, treated with RT alone at the same institutions from April 1994 to December 1996 (Table 2).

2.4. Statistical Analysis. Survival was calculated actuarially using the Kaplan Meier method, and significance was assessed using the log-rank test. Multivariate analysis by the Cox Regression Model was performed for identifying the independent prognostic variables governing the clinical end points.

The length of survival was considered from the end of radiation treatment until the last follow-up or the death.

\section{Results}

3.1. Survival. At the time of this analysis, May 2014, 3 pts (9.6\%) in group A with AA and 5 pts (15.1\%) in group B with AA are still alive, and 56 pts are dead (28 pts in group A and 28 pts in group B). The median follow-up in group A pts has been 18 months (range 6-89) and in group B 16 months (range $3-70)$.

On the basis of Kaplan Meiers estimates, the 1- and 2-year overall survival rates (OS) were, respectively, $74 \%$ and $29 \%$ in group A pts and $73 \%$ and $30 \%$ in group $B(P=0.8$ not statistically significant) (Figure 1).

On the contrary, a statistically significant better 2-year OS was observed in pts with age $\leq 55$ years $(P=0.04)$ and/or with diagnosis of AA $(P<0.0001)$ and/or with total dose delivered $\geq 60$ Gy $(P=0.001)$ (Table 4$)$.

The multivariate analysis, using stratified Cox regression, disclosed a significant better 2-year OS associated with age $\leq 55$ years $(P=0.04)$, diagnosis of AA $(P=0.0003)$, and type

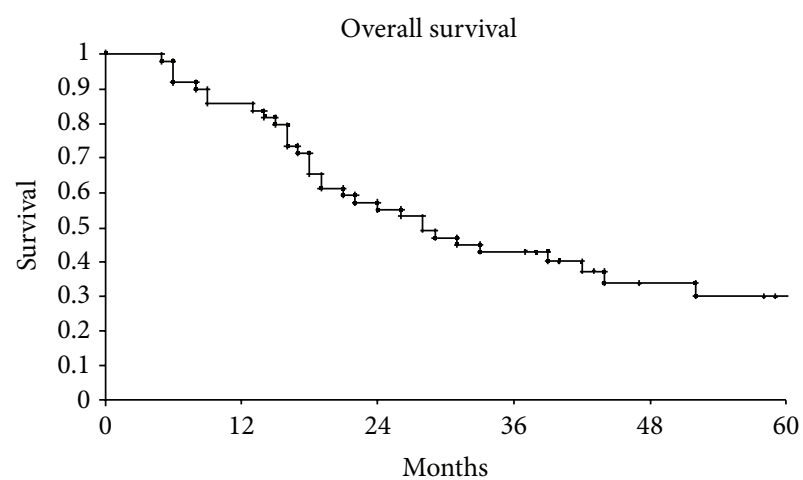

FIgURE 1: Overall survival of our series.

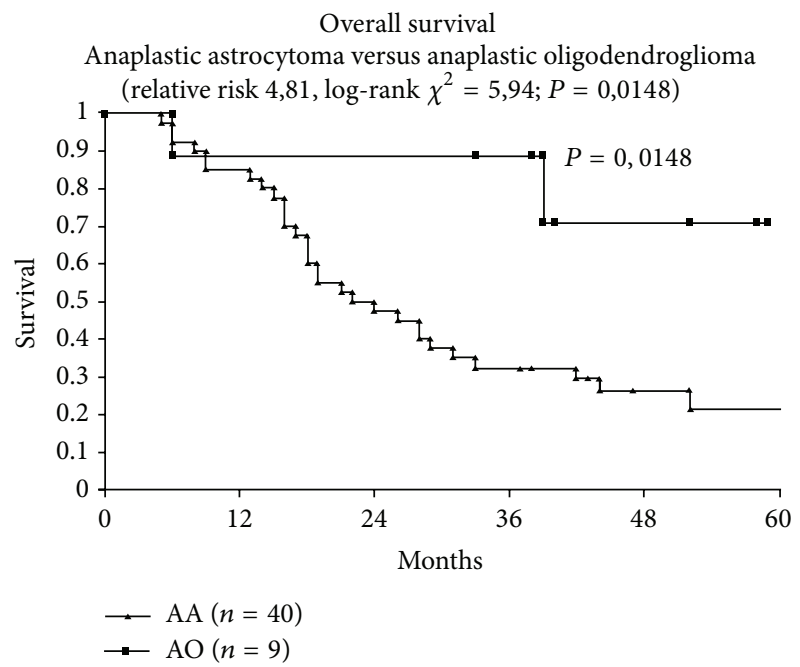

FIGURE 2: Overall survival anaplastic astrocytoma versus anaplastic oligodendroglioma.

of surgery $(P=0.05)$. Timing of TMZ administration (group A versus group B) was not statistically significant (Table 5).

Comparing the results of the 64 pts of groups $\mathrm{A}+\mathrm{B}$ versus the 64 pts of group $\mathrm{C}$, the median follow-up in groups $\mathrm{A}$ $+\mathrm{B}$ pts has been 17.5 months (range 3-89) and in group C pts 14 months (range 4-62). On the basis of Kaplan Meier estimates, the median OS was 15 months: 14 months in subjects not treated with TMZ (group C) and 17.5 months in the patients (groups $\mathrm{A}+\mathrm{B})$ that received TMZ $(P=0.0001)$ (Figure 2). Age, AA histology, and administration of TMZ were statistically significant prognostic factors for 2-year OS in the univariate analysis using Kaplan Meier method and compared with log-rank test: age $\leq 55$ years $P=0.007$; AA histology $P<0.0001$; administration of TMZ $P=0.0001$ (Table 6).

PFS was for GBM 9 months and for AA 11.

The salvage therapies employed in local recurrence are fotemustine, antiangiogenic drugs, and temozolomide.

3.2. Toxicity. We analysed complications of the 64 pts treated with RT and TMZ according to the WHO-RTOG scale. Grade 3 (G3) haematological toxicity was scored in 6 pts 
TABLE 3: G3 haematological toxicity in group A and group B patients.

\begin{tabular}{lcc}
\hline & Group A & Group B \\
\hline Patients' number & 31 & 33 \\
Haematological toxicity & $6.4 \%$ & $12.1 \%$ \\
Thrombocytopenia & $0 \%$ & $3 \%$ \\
Neutropenia & $3.2 \%$ & $6 \%$ \\
Thrombocytopenia-neutropenia & $3.2 \%$ & $3 \%$ \\
\hline
\end{tabular}

TABLE 4: Univariate analysis group A and group B patients.

\begin{tabular}{lccc}
\hline Prognostic factors & Pts' number & 2-year OS & $P$ \\
\hline Age & & & \\
$\quad \leq 55$ years & 26 & $38.4 \%$ & 0.04 \\
$\quad>55$ years & 38 & $23.7 \%$ & \\
Sex & & & \\
$\quad$ Male & 35 & $28.6 \%$ & n.s. \\
$\quad$ Female & 29 & $31 \%$ & \\
Histology & & & \\
$\quad$ Anaplastic astrocytoma & 21 & $52.4 \%$ & $<0.0001$ \\
$\quad$ Glioblastoma & 43 & $16.3 \%$ & \\
$\quad$ Multiforme & & & \\
Surgery & & & \\
$\quad$ Stereotactic biopsy & 12 & $25 \%$ & \\
$\quad$ Subtotal resection & 30 & $23.3 \%$ & n.s. \\
$\quad$ Total resection & 22 & $36.6 \%$ & \\
Timing TMZ & & & \\
$\quad$ Adjuvant & 31 & $29 \%$ & \\
$\quad$ Concomitant/adjuvant & 33 & $30 \%$ & \\
RT total dose & & & \\
$\quad \geq 60$ Gy & 54 & $33.3 \%$ & 0.001 \\
$\quad<60$ Gy & 10 & $10 \%$ & \\
\hline
\end{tabular}

( $9 \%$ of pts): 2 belonging to group A and 4 to group $\mathrm{B}(P=0.6$ not statistically significant) (Table 3 ).

In group A pts, during adjuvant chemotherapy, only one patient developed G3 neutropenia-thrombocytopenia and a further subject showed G3 neutropenia alone. No pts of group A experienced thrombocytopenia alone. In the subjects treated with concomitant TMZ and RT (group B) we observed G3 neutropenia in 2 cases, G3 thrombocytopenia in 1 , and neutropenia and thrombocytopenia in a further $r$ patient (Table 3).

No G4 haematological toxicity was observed.

The other acute side effects (G1-G2 nausea, vomiting, and fatigue), reported in 10 pts, of groups A + B were mild or easily controlled with medications.

\section{Discussion}

Malignant gliomas are among the most uncontrollable, devastating, and fatal cancers. The benefit of RT alone, in inoperable pts, or in combination with surgery, has been
TABLE 5: Multivariate analysis group A and group B patients.

\begin{tabular}{lc}
\hline Prognostic factors & $P$ \\
\hline Age & \\
$\quad \leq 55$ years- $>55$ years & 0.04 \\
Sex & n.s. \\
$\quad$ Male-female & \\
Histology & 0.0003 \\
$\quad$ AA—GBM & \\
Surgery & 0.05 \\
$\quad$ Stereotactic Biopsy-subtotal resection-total resection & \\
Timing TMZ & \\
$\quad$ Adjuvant-concomitant/adjuvant & n.s. \\
RT total dose & \\
$\quad<60$ Gy- $\geq 60$ Gy & 0.0001 \\
\hline
\end{tabular}

TABLE 6: Univariate analysis groups A + B and group C patients.

\begin{tabular}{lccc}
\hline Prognostic factors & Pts' number & 2-year OS & $P$ \\
\hline Age & & & \\
$\quad \leq 55$ years & 47 & $29 \%$ & 0.007 \\
$\quad>55$ years & 81 & $15 \%$ & \\
Sex & & & \\
$\quad$ Male & 74 & $17.5 \%$ & n.s. \\
$\quad$ Female & 54 & $22 \%$ & \\
Histology & & & \\
$\quad$ Anaplastic astrocytoma & 36 & $40 \%$ & $<0.0001$ \\
$\quad$ Glioblastoma & 92 & $11.8 \%$ & \\
$\quad$ multiforme & & & \\
Surgery & & & \\
$\quad$ Stereotactic biopsy & 23 & $25 \%$ & \\
$\quad$ Subtotal resection & 65 & $14 \%$ & \\
$\quad$ Total resection & 40 & $26 \%$ & \\
Treatment schedule & & & \\
$\quad$ RT + TMZ & 64 & $29.6 \%$ & 0.0001 \\
$\quad$ RT alone & 64 & $9.3 \%$ & \\
RT total dose & & & \\
$\quad \geq 60$ Gy & 109 & $22 \%$ & 0.003 \\
$\quad<60$ Gy & 19 & $10.5 \%$ & \\
\hline
\end{tabular}

demonstrated in phase III trials at the end of seventies [1114]. In order to improve the outcome, various combinations of surgery, RT, and chemotherapy have been tried in several studies, unfortunately with inconclusive results [1-6]. A meta-analysis [7] has pointed out significant improvement in survival adding nitrosourea based regimens.

TMZ is nowadays the first line chemotherapeutic drug in GBM therapy. Our study confirms its usefulness and the lack of heavy side effects.

Experimental studies demonstrated in vitro synergistic effect, in inhibiting glioblastoma cell lines growth, by using 
TMZ and fractionated RT [15]. On the basis of these suggestions, phase I and II clinical trials investigated the efficacy of this association, with promising results [16-24].

According to these encouraging experiences in 1997 we started to treat pts affected by high grade gliomas with a combination of TMZ and RT. The results of our study confirm the literature data regarding tolerability and usefulness of this schedule.

The main toxicity in our experience has been haematological, with G3 neutropenia, thrombocytopenia, or both observed in $9 \%$ of cases and above all in concomitant/adjuvant TMZ administration, without statistical significance. Similar incidence of haematological toxicity has been reported in Stupp phase II trial [22] and in other preliminary experiences $[16,25]$. In our series we observed neither G4 haematological side effects nor infections of Pneumocystis carinii $[17,22]$. Moreover nonhaematological toxicities were mild and easily controlled by medical therapies.

The median survival obtained in our pts is similar to that reported in other publications [26-30]. Two-year OS in groups $\mathrm{A}+\mathrm{B}$ pts was $29.6 \%$ but $9.3 \%$ in group $\mathrm{C}$ $(P=0.0001)$, suggesting a significant improvement of prognosis by combined treatment. Similar results, using RT and TMZ, have been shown in other phase II trials with 2year survival ranging from $29 \%$ to $38 \%$ [26-30]. Moreover in the multicentric randomized EORTC-NCIC 26981 trial 2year survival was $26 \%$ in the 287 pts of RT + TMZ arm versus $8 \%$ in the 286 pts of RT alone arm $(P<0.0001)$ [24, 31].

In our pts no statistically significant difference in OS between adjuvant and concomitant/adjuvant TMZ administration was observed. Anyway preclinical studies and larger clinical trials have suggested additive or perhaps synergistic activity combining TMZ and RT [22].

In agreement with literature $[22,32,33]$, our data confirm that age and histology represent important prognostic factors in this disease. In fact both univariate and multivariate analyses showed that pts with age $\leq 55$ years and diagnosis of anaplastic astrocytoma have a significantly better survival.

Improvement of prognosis, obtained using RT and TMZ in malignant gliomas in several phase II series and above all in the phase III EORTC-NCIC 26981 trial on GBM, suggests that actually this treatment can be used routinely in clinical practice [24].

Despite these interesting results the prognosis of malignant gliomas remains poor. Concerning this, great advances could come from research into genetic features of brain tumours, with the aim of characterising molecular profiles of neoplasm [34]. These developments will identify novel drug targets and therapeutic strategies, in order to individuate subgroups of pts receiving tailored treatments, on the basis of the genetic findings of their cancers [34]. According to these remarks, some recent reports show the preliminary results of combining TMZ with other drugs active against biological targets, particularly antiangiogenic drugs like thalidomide [35] and rofecoxib [36] and other proposed "old" drugs like metformin and arsenic trioxide [37].

In order to increase the efficacy of TMZ, Brock et al. [35] have employed in 67 pts with glioblastoma an association of TMZ, thalidomide, and RT. They observed an acceptable tolerance and a favourable survival outcome when compared with a historical group of pts treated with RT alone or RT and nitrosourea adjuvant chemotherapy.

Similar findings were reported by Baumann et al. [36] in a recent publication, whereas in a preliminary study TMZ was tested in pts with GBM in combination with the COX2 inhibitor rofecoxib, another antiangiogenic agent, in order to evaluate the safety and activity of this association [38].

Moreover several phase I and II trials are exploring possible therapeutic approaches with schedules containing TMZ and new drugs $[23,39,40]$.

Some authors believe that the additions of TMZ do not change the pattern of progression of GBM after radiotherapy (GUNJUR A. J. M. I. and RADIATION ONCOLOGY 2012).

On the contrary, the majority, considering that patients aged 75 or older represent half of all patients with GBM, retain that older cohort ( $>65$ years) should not be excluded from treatments as was shown in NOA- 8 phase III study; data from randomized and nonrandomized studies show encouraging results.

Finally, elderly patients will soon represent the vast majority of patients with GBM and they deserve to be treated at the best way possible; future studies should include the older patients with stratification of comorbidities and PS.

For what concerns anaplastic astrocytomas, the treatment of anaplastic glioma varies depending on histopathology of the tumor, molecular markers, and individual patient characteristics. As opposed to the standard treatment of glioblastoma, based on Stupp trial, there is no accepted standard treatment for AG. AA is most often treated with radiotherapy, with or without concomitant TMZ and with or without adjuvant temozolomide. Temozolomide has largely replaced PCV (procarbazine, CCNU, and vincristine) as the chemotherapeutic agent for $\mathrm{AO}$ and $\mathrm{AOA}$, largely due to greater tolerability and less potential for toxicity. However, whether temozolomide has similar efficacy to PCV has not been fully evaluated. Patients, who have progressed after RT alone, may be treated with TMZ or PCV. A valid option, at the recurrence, is stereotactic radiosurgery and we employ this modality in many patients.

In conclusion there is, today, an improvement in surgical techniques, such as fluorescence guided resection and neuroendoscopic approaches; new discoveries will be made in basic and translation research, with block of cancer proliferation (e.g., TMZ, BEVACIZUMAB, IHDAC, anti-P53 inhibitors, inhibition of cancer stem cells, more advanced and precise radiation techniques, inhibitors of EGFR, TKI, NF$\mathrm{KB}$, inhibitors of mTOR, Pi 3k/AKT, and proteasome) and new delivery of drugs in nanoparticles and liposomes and the introduction in clinical practice of antipsychotic drugs (like haloperidol of II and III generation). All that will, probably, improve survival and quality of life in such a devastating disease.

\section{Conclusions}

Continuous daily TMZ and concomitant RT followed by adjuvant TMZ are safe and can prolong survival in pts with 
high grade gliomas. Our report confirms the beneficial role of the association RT + TMZ on long ( $>10 \mathrm{y})$ follow-up. It must be stressed that this association resulted in life-saving on a 13year time span in 3 out the 21 patients (14.1\%) with anaplastic astrocytoma.

There is today a large interest in new treatments in gliomas like improvement in surgical techniques such as fluorescence guided resection and neuroendoscopic approaches. New discoveries are made in basic and translation research, and old and new drugs have been proposed as promising agents in brain tumors care [41-50]. More advanced and precise radiation techniques, inhibitors of EGFR, TKI, NF-KB, inhibitors of mTOR, Pi 3k/AKT, proteasome, and new delivery of drugs in nanoparticles, liposomes, and the introduction in clinical practice of antipsychotic drugs (like haloperidol of II and III generation) could be beneficial. All these improvements and developments will, probably, improve survival and quality of life in such a devastating disease.

\section{Conflict of Interests}

The authors declare that there is no conflict of interests regarding the publication of this paper.

\section{References}

[1] L. M. DeAngelis, P. C. Burger, S. B. Green, and J. G. Cairncross, "Malignant glioma: who benefits from adjuvant chemotherapy?” Annals of Neurology, vol. 44, no. 4, pp. 691-695, 1998.

[2] E. Galanis and J. Buckner, "Chemotherapy for high-grade gliomas," British Journal of Cancer, vol. 82, no. 8, pp. 1371-1380, 2000.

[3] J. Del Rowe, C. Scott, M. Werner-Wasik et al., "Single-arm, open-label phase II study of intravenously administered tirapazamine and radiation therapy for glioblastoma multiforme," Journal of Clinical Oncology, vol. 18, no. 6, pp. 1254-1259, 2000.

[4] H. A. Fine, K. B. G. Dear, J. S. Loeffler, P. M. Black, and G. P. Canellos, "Meta-analysis of radiation therapy with and without adjuvant chemotherapy for malignant gliomas in adults," Cancer, vol. 71, no. 8, pp. 2585-2597, 1993.

[5] B. J. Fisher, C. Scott, D. R. Macdonald, C. Coughlin, and W. J. Curran, "Phase I study of topotecan plus cranial radiation for glioblastoma multiforme: results of Radiation Therapy Oncology Group trial 9507," Journal of Clinical Oncology, vol. 19, no. 4, pp. 1111-1117, 2001.

[6] D. Thomas, M. Brada, S. Stenning et al., "Randomized trial of procarbazine, lomustine, and vincristine in the adjuvant treatment of high-grade astrocytoma: a Medical Research Council Trial," Journal of Clinical Oncology, vol. 19, no. 2, pp. 509-518, 2001.

[7] Glioma Meta-Analysis Trialist (GMT) Group, "Chemotherapy in adult high-grade glioma: a systematic review and metaanalysis of individual patient data from 12 randomised trials," The Lancet, vol. 359, no. 9311, pp. 1011-1018, 2002.

[8] R. Stupp, M. Gander, S. Leyvraz, and E. Newlands, "Current and future developments in the use of temozolomide for the treatment of brain tumours," The Lancet Oncology, vol. 2, no. 9, pp. 552-560, 2001.
[9] P. Corsa, S. Parisi, M. Piombino et al., "A preliminary retrospective study with temozolomide and radiotherapy versus radiotherapy alone for the treatment of high-grade gliomas," International Journal of Radiation Oncology, Biology, Physics, vol. 57, supplement 2, p. S378, 2003.

[10] E. P. M. Jansen, L. G. H. Dewit, M. Van Herk, and H. Bartelink, "Target volumes in radiotherapy for high-grade malignant glioma of the brain," Radiotherapy and Oncology, vol. 56, no. 2, pp. 151-156, 2000.

[11] A. P. Andersen, "Postoperative irradiation of glioblastomas. Results in a randomized series," Acta Radiologica: Oncology, vol. 17, pp. 474-484, 1978.

[12] K. Kristiansen, S. Hagen, T. Kollevold et al., "Combined modality therapy of operated astrocytomas grade III and IV. Confirmation of the value of postoperative irradiation and lack of potentiation of bleomycin on survival time: a prospective multicenter trial of the Scandinavian Glioblastoma Study Group," Cancer, vol. 47, no. 4, pp. 649-652, 1981.

[13] W. R. Shapiro and D. F. Young, "Treatment of malignant glioma. A controlled study of chemotherapy and irradiation," Archives of Neurology, vol. 33, no. 7, pp. 494-500, 1976.

[14] M. D. Walker, E. Alexander Jr., W. E. Hunt et al., "Evaluation of BCNU and/or radiotherapy in the treatment of anaplastic gliomas. A cooperative clinical trial," Journal of Neurosurgery, vol. 49, no. 3, pp. 333-343, 1978.

[15] J. Van Rijn, J. J. Heimans, J. Van Den Berg, P. Van Der Valk, and B. J. Slotman, "Survival of human glioma cells treated with various combination of temozolomide and X-rays," International Journal of Radiation Oncology Biology Physics, vol. 47, no. 3, pp. 779-784, 2000.

[16] M. Brada, K. Hoang-Xuan, R. Rampling et al., "Multicenter phase II trial of temozolomide in patients with glioblastoma multiforme at first relapse," Annals of Oncology, vol. 12, no. 2, pp. 259-266, 2001.

[17] S. M. Chang, K. R. Lamborn, M. Malec et al., "Phase II study of temozolomide and thalidomide with radiation therapy for newly diagnosed glioblastoma multiforme," International Journal of Radiation Oncology, Biology, Physics, vol. 60, no. 2, pp. 353-357, 2004.

[18] O. L. Chinot, S. Honore, H. Dufour et al., "Safety and efficacy of temozolomide in patients with recurrent anaplastic oligodendrogliomas after standard radiotherapy and chemotherapy," Journal of Clinical Oncology, vol. 19, no. 9, pp. 2449-2455, 2001.

[19] H. S. Friedman, R. E. McLendon, T. Kerby et al., "DNA mismatch repair and O6-alkylguanine-DNA alkyltransferase analysis and response to temodal in newly diagnosed malignant glioma," Journal of Clinical Oncology, vol. 16, no. 12, pp. 38513857, 1998.

[20] M. Gilbert, J. Olson, and W. Yung, "Preradiation treatment of newly diagnosed anaplastic astrocytomas and glioblastoma multiforme using temozolomide," Neuro-Oncology, vol. 2, abstract 77, p. 264, 2000.

[21] E. S. Newlands, M. F. G. Stevens, S. R. Wedge, R. T. Wheelhouse, and C. Brock, "Temozolomide: a review of its discovery, chemical properties, pre-clinical development and clinical trials," Cancer Treatment Reviews, vol. 23, no. 1, pp. 35-61, 1997.

[22] R. Stupp, P.-Y. Dietrich, S. O. Kraljevic et al., "Promising survival for patients with newly diagnosed glioblastoma multiforme treated with concomitant radiation plus temozolomide followed by adjuvant temozolomide," Journal of Clinical Oncology, vol. 20, no. 5, pp. 1375-1382, 2002. 
[23] B. Yamini, X. Yu, G. Y. Gillespie, D. W. Kufe, and R. R. Weichselbaum, "Transcriptional targeting of adenovirally delivered tumor necrosis factor $\alpha$ by temozolomide in experimental glioblastoma," Cancer Research, vol. 64, no. 18, pp. 6381-6384, 2004.

[24] R. Stupp, W. P. Mason, M. J. van den Bent et al., "Radiotherapy plus concomitant and adjuvant temozolomide for glioblastoma," The New England Journal of Medicine, vol. 352, no. 10, pp. 987-996, 2005.

[25] W. K. A. Yung, R. E. Albright, J. Olson et al., "A phase II study of temozolemide vs. procarbazine in patients with glioblastoma multiforme at first relapse," British Journal of Cancer, vol. 83, no. 5, pp. 588-593, 2000.

[26] H. Athanassiou, M. Synodinou, E. Maragoudakis et al., "Randomized phase II study of temozolomide and radiotherapy compared with radiotherapy alone in newly diagnosed glioblastoma multiforme," Journal of Clinical Oncology, vol. 23, no. 10, pp. 2372-2377, 2005.

[27] S. Chibbaro, L. Benvenuti, A. Caprio et al., "Temozolomide as first-line agent in treating high-grade gliomas: phase II study," Journal of Neuro-Oncology, vol. 67, no. 1-2, pp. 77-81, 2004.

[28] L. Cionini, A. Tagliagambe, L. Fatigante et al., "A phase 2 study with concurrent and sequential temozolomide combined with radiation therapy in high grade gliomas," International Journal of Radiation Oncology * Biology * Physics, vol. 51, supplement 3, p. S206, 2001.

[29] S. E. Combs, S. Gutwein, D. Schulz-Ertner et al., "Phase I/IIstudy of temozolomide combined with radiation as postoperative treatment in primary glioblastoma multiforme," International Journal of Radiation Oncology, Biology, Physics, vol. 60, supplement 1, pp. S258-S259, 2004.

[30] G. Lanzetta, C. Campanella, A. Rozzi et al., "Temozolomide in radio-chemotherapy combined treatment for newly-diagnosed glioblastoma multiforme: phase II clinical trial," Anticancer Research, vol. 23, no. 6, pp. 5159-5164, 2003.

[31] R. O. Mirimanoff, W. Mason, R. Kortmann et al., "Radiotherapy (RT) and concomitant and adjuvant Temozolomide (TMZ) versus Radiotherapy alone for newly diagnosed glioblastoma (GBM): overall results and Recursive Partitioning Analysis (RPA) of a phase III randomized trial of the EORTC Brain Tumor and Radiotherapy Groups and NCIC Clinical Trial Group," International Journal of Radiation Oncology, Biology, Physics, vol. 60, supplement 1, p. S162, 2004.

[32] W. J. Curran Jr., C. B. Scott, J. Horton et al., "Recursive partitioning analysis of prognostic factors in three radiation therapy oncology group malignant glioma trials," Journal of the National Cancer Institute, vol. 85, no. 9, pp. 704-710, 1993.

[33] C. B. Scott, C. Scarantino, R. Urtasun et al., "Validation and predictive power of Radiation Therapy Oncology Group (RTOG) recursive partitioning analysis classes for malignant glioma patients: a report using RTOG 90-06," International Journal of Radiation Oncology Biology Physics, vol. 40, no. 1, pp. 51-55, 1998.

[34] D. R. Macdonald, "New frontiers in the treatment of malignant glioma," Seminars in Oncology, vol. 30, no. 6, pp. 72-76, 2003.

[35] C. S. Brock, E. S. Newlands, S. R. Wedge et al., "Phase I trial of temozolomide using an extended continuous oral schedule," Cancer Research, vol. 58, no. 19, pp. 4363-4367, 1998.

[36] F. Baumann, M. Bjeljac, S. S. Kollias et al., "Combined thalidomide and temozolomide treatment in patients with glioblastoma multiforme," Journal of Neuro-Oncology, vol. 67, no. 1-2, pp. 191-200, 2004.
[37] M. Carmignani, M. Aldea, O. Soritau et al., "Glioblastoma stem cells: a new target for metformin and arsenic trioxide," Journal of Biological Regulators and Homeostatic Agents, vol. 2, pp. 1-15, 2014.

[38] J. Tuettenberg, R. Grobholz, T. Korn, F. Wenz, R. Erber, and P. Vajkoczy, "Continuous low-dose chemotherapy plus inhibition of cyclooxygenase- 2 as an antiangiogenic therapy of glioblastoma multiforme," Journal of Cancer Research and Clinical Oncology, vol. 131, no. 1, pp. 31-40, 2005.

[39] M. Bartolomei, C. Mazzetta, D. Handkiewicz-Junak et al., "Combined treatment of glioblastoma patients with locoregional pre-targeted ${ }^{90} \mathrm{Y}$-biotin radioimmunotherapy and temozolomide," Quarterly Journal of Nuclear Medicine and Molecular Imaging, vol. 48, no. 3, pp. 220-228, 2004.

[40] R. Ravizza, E. Cereda, E. Monti, and M. B. Gariboldi, "The piperidine nitroxide Tempol potentiates the cytotoxic effects of temozolomide in human glioblastoma cells," International Journal of Oncology, vol. 25, no. 6, pp. 1817-1822, 2004.

[41] P. Corsa, S. Parisi, A. Raguso et al., "Temozolomide and radiotherapy as first-line treatment of high-grade gliomas," Tumori, vol. 92, no. 4, pp. 299-305, 2006.

[42] C. A. Barker, A. J. Bishop, M. Chang, K. Beal, and T. A. Chan, "Valproic acid use during radiation therapy for glioblastoma associated with improved survival," International Journal of Radiation Oncology Biology Physics, vol. 86, no. 3, pp. 504-509, 2013.

[43] G. D. Guthrie and S. Eljamel, "Impact of particular antiepileptic drugs on the survival of patients with glioblastoma multiforme," Journal of Neurosurgery, vol. 118, no. 4, pp. 859-865, 2013.

[44] P. Evers, P. P. Lee, J. DeMarco et al., "Irradiation of the potential cancer stem cell niches in the adult brain improves progressionfree survival of patients with malignant glioma," BMC Cancer, vol. 10, article 384, 2010.

[45] H. C. Tsai, K. C. Wei, C. N. Tsai et al., "Effect of valproic acid on the outcome of glioblastoma multiforme," British Journal of Neurosurgery, vol. 26, no. 3, pp. 347-354, 2012.

[46] S. Scoccianti, S. M. Magrini, U. Ricardi et al., "Patterns of care and survival in a retrospective analysis of 1059 patients with glioblastoma multiforme treated between 2002 and 2007: a multicenter study by the central nervous system study group of Airo (Italian association of radiation oncology)," Neurosurgery, vol. 67, no. 2, pp. 446-458, 2010.

[47] T. Iuchi, K. Hatano, T. Kodama et al., "Phase 2 trial of hypofractionated high-dose intensity modulated radiation therapy with concurrent and adjuvant temozolomide for newly diagnosed glioblastoma," International Journal of Radiation Oncology Biology Physics, vol. 88, no. 4, pp. 793-800, 2014.

[48] S. Scoccianti, S. M. Magrini, U. Ricardi et al., "Radiotherapy and temozolomide in anaplastic astrocytoma: a retrospective multicenter study by the Central Nervous System Study Group of AIRO (Italian Association of Radiation Oncology)," NeuroOncology, vol. 14, no. 6, pp. 798-807, 2012.

[49] T. S. Armstrong, J. S. Wefel, M. Wang et al., "Net clinical benefit analysis of radiation therapy oncology group 0525: a phase III trial comparing conventional adjuvant temozolomide with dose-intensive temozolomide in patients with newly diagnosed glioblastoma," Journal of Clinical Oncology, vol. 31, no. 32, pp. 4076-4084, 2013.

[50] A. Gunjur, M. Bressel, and G. Ryan, "The addition of temozolomide does not change the pattern of progression of glioblastoma multiforme post-radiotherapy," Journal of Medical Imaging and Radiation Oncology, vol. 56, no. 5, pp. 567-573, 2012. 

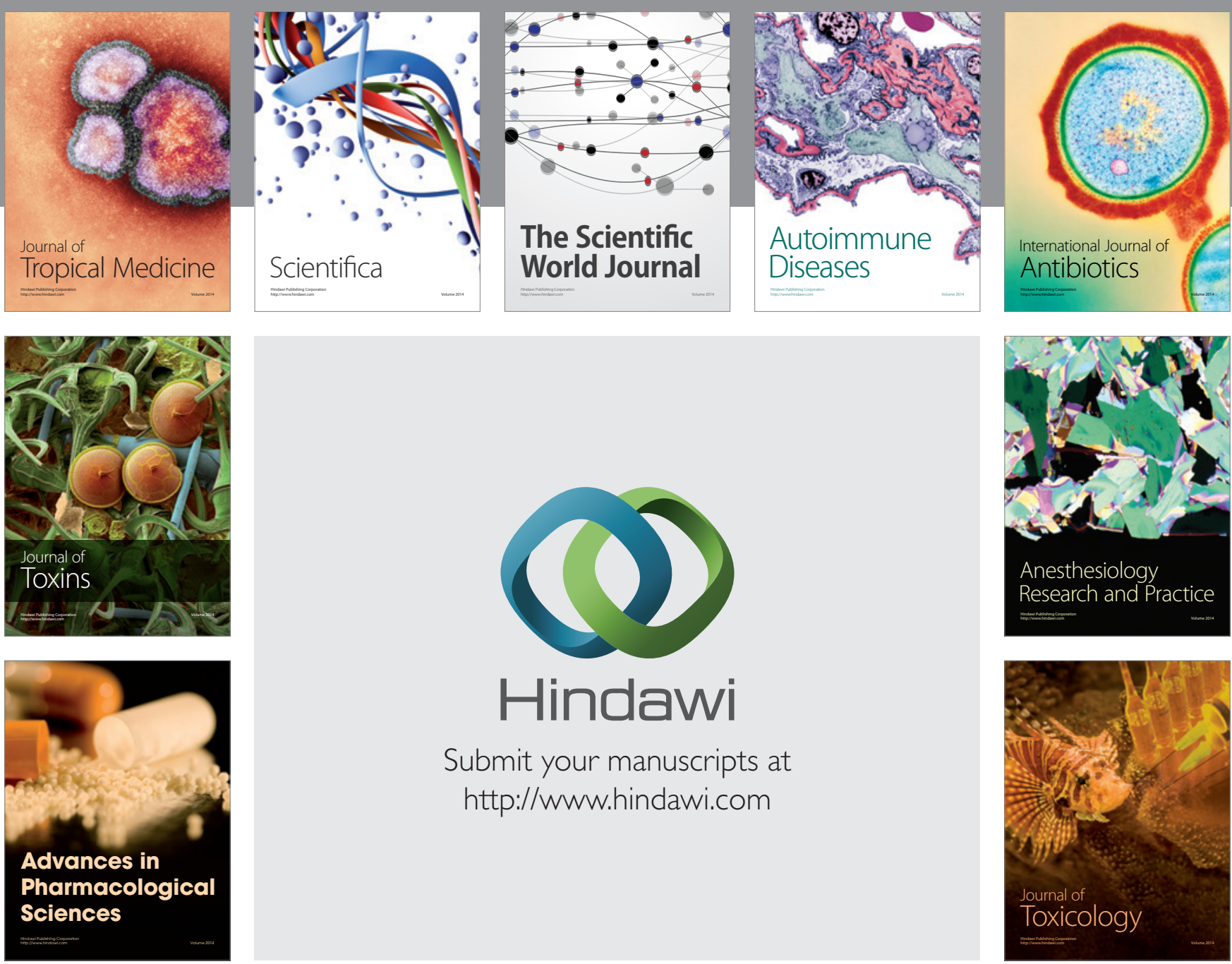

\section{Hindawi}

Submit your manuscripts at

http://www.hindawi.com
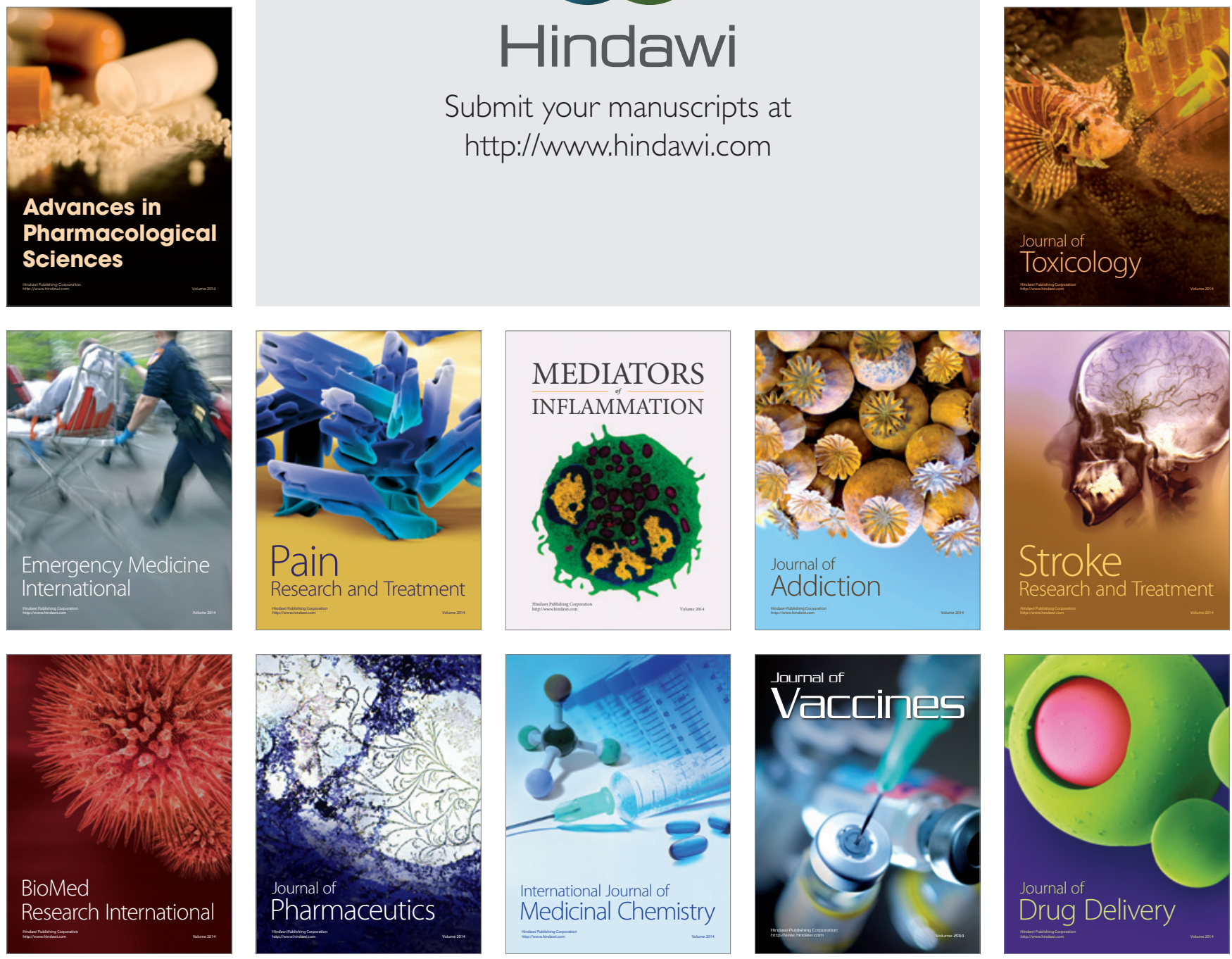